\title{
SciBX
}

\section{Targeting viral capsid assembly}

\section{By Tracey Baas, Senior Editor}

Researchers at Prosetta Antiviral Inc. have used an in vitro screen to identify small molecules targeting the host-catalyzed capsid assembly pathway for the rabies virus. ${ }^{1}$ The biotech has partnered with BristolMyers Squibb Co. to use the screen to identify small molecules targeting the pathway for HIV.

The viral capsid is a multiprotein complex that gives a virus its shape and protects its genome. Although once thought to form spontaneously, the viral capsid has recently been shown by Jaisri Lingappa and others to form via a pathway catalyzed by a host assembly complex. ${ }^{2-4}$ Thus, directly targeting the proteins comprising the host assembly complex could be a way to block formation of the viral capsid and prevent viral infection.

Lingappa is coauthor of the paper. She is an associate professor in global health and an adjunct associate professor in medicine and microbiology at the University of Washington.

In the new work, Prosetta used its "Our goal is to advance
one compound that
inhibits the capsid
formation of all viruses
responsible for human
respiratory disease."
- Vishwanath Lingappa,
Prosetta Antiviral Inc. previously developed cell-free protein synthesis (CFPS) screen to identify compounds that blocked virus capsid assembly (see Figure 1, "Proposed rabies capsid-assembly pathway"). The screen consisted of host cell extract, which included the host assembly proteins, and three capsid proteins from the rabies virus. Using the system, the team identified a small set of active antiviral compounds.

Prosetta's collaborators at the Centers for Disease Control and

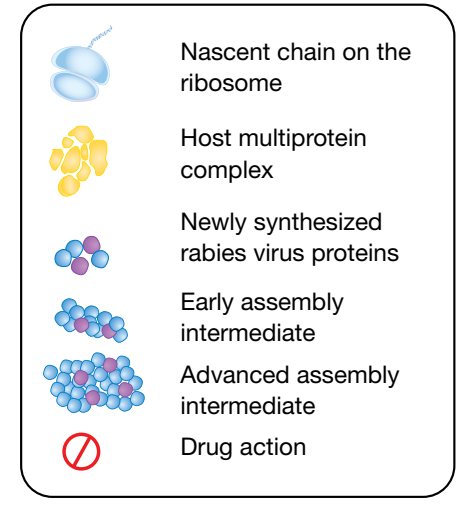

Figure 1. Proposed rabies capsidassembly pathway. Rabies viral proteins are translated by the ribosome (I[a]). The rabies phosphoprotein (blue) associates with unassembled nucleoprotein monomers (I[b]). One (or more) host multiprotein complex then catalyzes construction of the virus capsid through discrete assembly intermediates (I[c]) to provide the complete capsid (I[d]).

The cell-free protein synthesis (CFPS) whole-pathway screen (II) contains cellular extract, rabies nucleoprotein, matrix protein and phosphoprotein mRNAs, amino acids and an energyregenerating system (II[a]). The proposed synthesis and assembly of rabies capsid proteins occurs as described in (I) (II $[\mathbf{b}(1)])$. The CFPS translation products are transferred to a capture plate coated with anti-rabies nucleoprotein antibody. The captured rabies assembly intermediates bind a biotinylated secondary antibody (II[c(1)]), which is used to produce a fluorescent readout that indicates capsid assembly (II[d(1)]).

The CFPS system components are combined, and the drug is added $\leq$ six hours later (II[a]). A drug that blocks the formation of large capsid multimers (II[b(2)]) will lead to the capture plate being coated with monomeric units of rabies proteins (II[c(2)]) and inhibition of the fluorescent readout (II[d(2)]). (Figure based on Figures 2 and 3 from ref. 1.) 
Prevention next evaluated the compounds against a strain of 'street rabies' isolated from a rabid gray fox. In a rabies-infected mammalian cell culture model, an optimized lead molecule showed an $\mathrm{EC}_{50}$ value of 15-30 nM and a 50\% cytotoxicity concentration $\left(\mathrm{CC}_{50}\right)$ value of about 2.5-10 $\mu \mathrm{M}$.

The Prosetta team then used the small molecule in affinity chromatography to isolate the host capsid assembly machinery, a multiprotein complex. Detailed analysis of the complex showed that, among other proteins, it also contained ATP-binding cassette subfamily E member 1 (ABCE1), which had been previously associated with HIV capsid assembly.

Results were published in the Proceedings of the National Academy of Sciences.

"Going after a multicomponent host assembly machine requires a different way to think about screening," said Anna Mapp, professor of chemistry and director of the program in chemical biology at the University of Michigan and research professor at the university's Life Sciences Institute.

"Traditional screening methodologies look for high-affinity compounds to bind to small surfaces that participate in proteinprotein interactions or aim at enzymatic components of a complex. Prosetta's technology provides a way to identify hits based on function-in this case, disrupting the virus life cycle by impeding capsid formation-irrespective of target," explained Mapp.

\section{Clarity for the capsid}

Prosetta's next steps toward the ultimate development of these compounds as drugs should be "to test the small set of compounds in more cell types and animals and also against different viral strains for breadth of effect," said Stephen Goff, professor of microbiology and immunology and of biochemistry and molecular biophysics at Columbia University. "I'd also like details about the mechanism of action of the candidate lead for the target. What components are required, what directly binds the drugs and is the drug really impeding capsid formation?"

Vishwanath Lingappa, CTO and co-CEO of Prosetta, told SciBX that mechanistic studies to understand the compounds' mode of action are under way at Prosetta. The company's main focus, however, is to test the hits in animal models of rabies infection and continue using the screening platform to identify compounds that inhibit other viral infections, he said.
"We have already developed screens for 20 of the 23 virus families that cause human disease and have lead candidates targeting 14 different virus families," he said.

"One of our main projects is to find compounds that inhibit the capsid formation of multiple respiratory viruses. Right now, we have a subset of compounds that inhibits the six different virus families responsible for viral respiratory infection," said Vishwanath Lingappa. "Our goal is to advance one compound that inhibits the capsid formation of all viruses responsible for human respiratory disease."

In order to optimize the compound, he added, "we have functional assays in the form of cell-free assembly of the capsid via the hostcatalyzed pathway for each viral family, so we can use the activity in that assay to track the SAR and simply corroborate improvements in activity with improvements in activity against the real virus."

Prosetta has filed patent applications for the small molecules and the screening strategy.

Prosetta and Bristol-Myers Squibb (BMS) entered into a multiyear collaboration in July 2012 that includes a research program to discover and advance compounds shown to block HIV capsid assembly using the screening platform.

BMS has the right to develop and commercialize products arising from the research program. Prosetta will receive an upfront payment and multiyear research funding, and the biotech is eligible to receive milestone payments and royalties based on worldwide sales of drugs emerging from the collaboration.

BMS declined requests for interviews.

Prosetta is in discussions with two other undisclosed pharmas for partnering on different viral indications.

Baas, T. SciBX 6(8); doi:10.1038/scibx.2013.180

Published online Feb. 28, 2013

\section{REFERENCES}

1. Lingappa, U.F. et al. Proc. Natl. Acad. Sci. USA; published online Feb. 11, 2013; doi:10.1073/pnas.1210198110

Contact: Vishwanath R. Lingappa, Prosetta Antiviral Inc., San Francisco, Calif. e-mail: vlingappa@prosetta.com

2. Klein, K.C. et al. J. Virol. 85, 7419-7435 (2011)

3. Reed, J.C. et al. J. Cell Biol. 198, 439-456 (2012)

4. Klein, K.C. et al. J. Virol. 79, 6814-6826 (2005)

\section{COMPANIES AND INSTITUTIONS MENTIONED}

Bristol-Myers Squibb Co. (NYSE:BMY), New York, N.Y. Centers for Disease Control and Prevention, Atlanta, Ga. Columbia University, New York, N.Y. Prosetta Antiviral Inc., San Francisco, Calif. University of Michigan, Ann Arbor, Mich. University of Washington, Seattle, Wash. 